\section{Beyond the molecule}

\author{
Harry L. Anderson
}

Supramolecular Science. Editor-in-chief Wolfgang Knoll. Butterworth-Heinemann. 6/yr. \$254, £170 (institutional).

SUPRAMOLECULAR chemistry proclaims that there is a chemistry beyond molecular chemistry: molecules can be programmed to organize themselves into supramolecular assemblies that are more than the sum of their parts. Over the past 20 years, the structures studied by supramolecular chemists have expanded into the realms of nanotechnology. It has become clear that understanding how to design small molecular components so that they self-assemble into functional supramolecular architectures is fundamental to both molecular biology and materials science; this area is highly interdisciplinary.

Supramolecular Science was launched to provide an interdisciplinary forum for papers in the emerging fields of nanotechnology, supramolecular assemblies and 'smart' materials. The editor-in-chief,
Wolfgang Knoll, identified a need to collect contributions previously published in many different journals with little overlap in readership. The journal is extremely broad in its scope. It aims to publish original peer-reviewed articles covering chemical design of functional materials, physical principles of self-assembly, biological approaches to intelligent materials, molecular engineering fabrication techniques, new techniques for structure determination at the molecular level and computational methods for predicting the properties of supramolecular aggregates. Each issue consists of about six original articles (short communications and full papers) and one "laboratory profile". These profiles are a refreshing change from the standard review format.

The journal has succeeded in attracting some high-quality papers, particularly in the areas of surface chemistry and colloids. But its broad scope means that it does not have a well-defined niche and I doubt whether it can compete with journals such as Langmuir and Advanced Materials which cover a similar area.

Harry L. Anderson is at the Dyson Perrins Laboratory, University of Oxford, South Parks Road, Oxford OX1 3QY, UK.

\title{
Independence play
}

\section{Dave Cliff}

Autonomous Robots. Editor George A. Bekey. Kluwer. 4/yr. DFI468, \$268 (institutional); DFI250, $\$ 105$ (personal).

Although malignant androids with human-level intelligence are likely always to be fiction, they have one property that it is both desirable and realistic to attempt to build into future models of industrial or commercial robots: autonomy. Like familiar industrial robots, an autonomous robot has sensors (for example, video cameras) and actuators (for example, motors); but,

IMAGE
UNAVAILABLE
FOR COPYRIGHT
REASONS

\section{For freewheelers and wannabees}

\section{Ian Stewart}

\section{Complexity. Editor-in-chief Harold} Morowitz. Wiley. 6/yr. USA \$195, elsewhere \$225 (institutional); USA \$69.95, elswhere $\$ 89.95$ (personal); USA $\$ 36$, elsewhere $\$ 66$ (student).

I AM an unabashed fan of complexity theory, although not of the belief that it has all the answers, and I am far from convinced that its current methodology is anything other than a convenient stopgap. When we really start to understand how high-level structure emerges from lowlevel rules in complex systems, we will surely rely less on simulations and more on conceptual insights. But if we are ever to reach this happy state then the burgeoning activity in the area needs a focus. Complexity is the first and only specialist journal: how effective a focus is it?

Its academic pedigree is impeccable. Production quality is terrific: it looks like a newsstand magazine. Its contents are organized like a magazine too: editorial material, tutorial articles and surveys outweigh specialist papers. It is not a bad way to organize an interdisciplinary journal. Specialist articles ought to be able to survive in journals appropriate to their own applied area, and will be far more widely read there than in a ghetto publication but where can you read or publish free- wheeling discussions of broader issues?

The variety of contributions is wonderful: linguistic patterns, genetic algorithms, financial markets, technological evolution, phase transitions as universal principles, artificial intelligence, cellular automata, order and disorder in ant colonies. The first few issues are perhaps a bit too self-conscious, but it is not easy to start up something of this kind without some backward glances. This is a house journal for complexity theorists and wannabees, with plenty to offer any interested scientist or mathematician who has the inclination and imagination to ask the hard questions.

As Harold Morowitz says in a perceptive opening editorial, the hierarchical organization of science has given us a deep understanding of many levels of the Universe, from atoms to ecosystems, but what it has not given us is any comparable understanding of the links from one level to the next. The world is full of scientists who confidently assert that these links exist, but few can offer any serious insights into what is actually going on. If you think such questions are worthy of serious effort, Complexity is for you.

Ian Stewart is in the Department of Mathematics, University of Warwick, Coventy CV4 $7 A L, U K$.

\section{Getting to grips with robotics.}

crucially, it is responsible for achieving desired tasks by coordinating its own perceptions and actions for extended periods of time without human intervention. Autonomous robots are typically mobile and self-sufficient. The ability to function independently, reacting appropriately to unpredictable or changing environments, opens up several important areas of application and presents many challenging research issues.

Unlike most established robotics journals, this new one is devoted solely to autonomous robots. Its stated aims and scope include robots that display mobility on land, under water, in the air or in space; the mobility may be provided by wheels, legs, fins, rotors or other propulsion devices. Such mobility requires research in sensing, in computer learning, control and adaptation, and in self-calibration and selfrepair. There is only one journal I know of that has a similar emphasis on autonomy, Robotics and Autonomous Systems.

Whether there is sufficient interest to sustain two journals remains to be seen. Certainly Autonomous Robots has several strong points in its favour. The 30 -member international editorial board includes many of the people who helped to shape the 